\title{
Prometheus Framework for Fuzzy Information Retrieval in Semantic Spaces
}

\author{
A. Andrushevich ${ }^{1}$, M. Fercu ${ }^{1}$, J. Hopf ${ }^{1}$, E. Portmann ${ }^{2}$ and A. Klapproth ${ }^{1}$ \\ ${ }^{1}$ CEESAR - iHomeLab, Lucerne University of Applied Sciences and Arts, Horw, Switzerland, \\ \{aliaksei.andrushevich, michael.fercu, joern.hopf, alexander.klapproth\}@hslu.ch \\ ${ }^{2}$ Information Systems Research Group, University of Fribourg, Fribourg, Switzerland, \\ edy.portmann@unifr.ch
}

\begin{abstract}
This paper introduces a novel vision for further enhanced Internet of Things services. Based on a variety of data (such as location data, ontologybacked search queries, in- and outdoor conditions) the Prometheus framework is intended to support users with helpful recommendations and information preceding a search for context-aware data. Adapted from artificial intelligence concepts, Prometheus proposes user-readjusted answers on umpteen conditions. A number of potential Prometheus framework applications are illustrated. Added value and possible future studies are discussed in the conclusion.
\end{abstract}

\section{Introduction and related work}

In the epigrammatic triumphant history of the Internet, first the World Wide Web was created as a CERN-project initiated by Timothy Berners-Lee. In the early Web, retrospectively referred to as Web 1.0, a small number of so-called information producers published their insights as a collection of static HTML pages and a great mass of consumers was opposed to these insights.

In the late 90s DiNucci first mentioned the term Web 2.0 and thus caused the advent of a new slogan. Afterwards O'Reilly declared that Web 2.0 technically did not differ from the earlier Web 1.0. In contrast to static expert-generated content, interactive elements are crucial in Web 2.0. Ever since the first use of the term Web 2.0 Berners-Lee deplored it as a marketing buzzword. He tried instead to advertise his future visions of the WWW with his ideas about the Semantic Web. The Semantic Web is an emerging development of the Internet in which not only the meaning or semantics of information is defined but also services on the Web, making it possible for machines to understand and satisfy the requests of both people and machines. Because of the enhancement to a machine-understandable Internet, the Semantic Web is sometimes called Web 3.0. Berners-Lee specified 
the Semantic Web as a component of Web 3.0.

In fall 2009, O'Reilly and Battele went further deeper by defining another upcoming buzzword "Web Squared" where the Web no longer is a collection of static pages that describe something in the world. Instead they outline in [O'Reilly and Battelle 2009] the Internet of Things. The Internet of Things exemplifies ubiquitous computing and "things that think". It describes a form of physical computing and is a non-deterministic, open network in which self-organized or intelligent entities will be interoperable and able to act independently - pursuing their own or shared objectives - depending on the context, circumstances or environment as described in [IoT Summary 2005].

These networks are delineated as ubiquitous computing models, which are post-desktop models of human-computer interaction, considered as an advancement from the desktop paradigm. However, when Web meets the world the vast data produced are mostly stored or provided in an unstructured way distributed on different systems; globally considered. An important case in ubiquitous computing for this reason is to find relevant information.

To find context relevant information in connection to real-world human-settledenvironment services, processes and systems become more crucial. A viable way to improve existing searches and to approach a universal Semantic Web (that is virtual Internet together with the real Internet of Things) is to teach the Web based on an automatically built ontology the meaning of real world parameter values. Additionally machine learning, a scientific discipline that is concerned with the design and development of algorithms, can be used to learn based on sensor and/or Internet data as Bishop elucidates in [Bishop 2008]. After Kasabov approaches to ML are expert systems whereby "an expert system is a program that can provide expertise for solving problems in a defined application area in the way the experts do" as explained in [Kasabov 1996].

To present expert-system-based real-time information in a clever way and to force the users to interact with the information, real and virtual worlds can melt into a new augmented reality. Thus augmented reality can be considered as an event of ubiquitous computing where virtual computer-generated symbolisms are superimposed into physical real-world environments, creating a mixed reality as Azuma et al. explain in [Azuma et al. 2001].

In Greek mythology, Prometheus (Ancient Greek for forethought) was a champion of humankind known for his intellect. He is said to be the benefactor of culture and the great instructor of all human beings. The ambitious project's goal, named after this transcendent ideal, is to offer the human race further techniques to master their human duties and responsibilities in an easier way by pooling virtual and real world aspects. 


\section{Applications of Fuzzy Sets Theory}

This section aims to introduce some concepts of fuzziness which deals with vague reasoning. To emphasize the benefits that fuzziness brings to artificial intelligence the first section 2.1 brings the affinity of human thinking and fuzziness in. Section 2.2 introduces fuzzy set theory and classification and section 2.3 fuzzy expert systems.

\subsection{Fuzziness and the Human Factor}

Mentioned in [Zadeh 1965] inter alia, fuzzy logic - a particular type of multivalued logic emerged as a corollary of Zadeh's proposition of fuzzy set theoryfollows the way humans think and helps to better handle real world facts, since human reasoning is undichotomic, contrasting computers, where all is either true (1) or false (0). It deals with haziness and the conceptions are polysemous in terms of that they cannot be sharply defined. Fuzzy logic brings imprecise human facts over to accurate mathematical models.

While variables in mathematics usually take numerical results, in fuzzy logic, the non-numeric linguistic variables are often used to cultivate the locution of rules and facts. A linguistic variable such as "size" can have a value just like 'tall' or its antonym 'short'. However, the great utility of linguistic variables is that they can be modified through linguistic transformation which can be associated with given functions. The question whether a person is 'tall' cannot be unmistakably answered, because it is not possible to clearly state if a person is 'tall'. An answer may depend on individual cognition and further for the individual itself it may even not be feasible to give a strict answer for the simple reason that belonging to a set (e.g. size) is often not sharp but fuzzy, involving a partial matching expressed in the natural language by the expressions 'quite', 'slightly', 'more or less', etc.

Figure 1 shows a tender varying curved line that passes gently from 'not-tall' to 'tall'. Therefore this line stipulates the transition of the linguistic variable "size". Both people are 'tall' to some degree (as both people are 'short' to some degree) but the female is significantly 'less tall' than the male. The vertical axis is an index reputed with the membership value between 0 and 1 ; the curve is noted as membership function.

\subsection{Introduction to fuzzy set theory and classification}

Fuzzy sets are an extension of the classical sets and incorporate special membership levels. In classical set theory, the membership of elements in a set is assessed in binary terms according to a two-valued condition; an element either belongs or doesn't belong to the set. By contrast, fuzzy set theory permits the gradual as- 
sessment of the membership of elements in a set; this is described by dint of a membership function valued in the real unit interval [0..1]. Therefore fuzzy sets generalize classical crisp sets, since the indicator functions of classical sets are special cases of the membership functions of fuzzy sets.

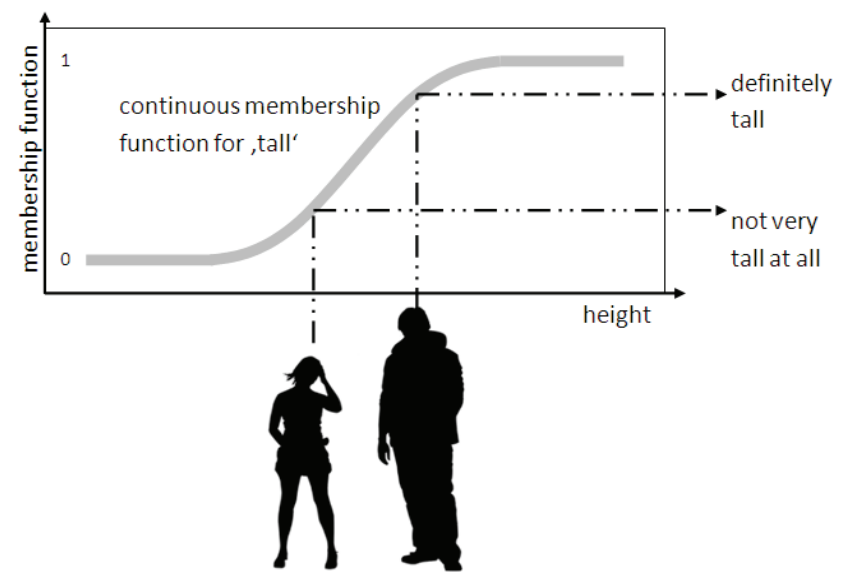

Fig. 1 The fuzzy height set illustrate the continuous membership function for the linguistic variable 'tall'

Fuzzy classification is an upgrading of traditional classification; equally fuzzy sets extend classical sets. The term classification describes the way of clumping elements into clusters, so that elements in the same cluster are as identical as possible, and elements in different clusters are as diverse as possible. In sharp classification each element is associated with just one cluster; as a result the belonging of the elements to clusters are reciprocal and exclusive. On the other hand fuzzy classification allows elements to belong to several clusters at the same time; and again like fuzzy sets, each element has a membership degree which reveals how far it belongs to the various clusters. Thereto fuzzy clustering algorithms allow the modeling of uncertainty associated with vagueness and imprecision and putting this into mathematical equations as described in [Portmann and Meier 2010]. In general fuzzy clustering algorithms a fuzzy cluster is represented by a representative element (typically the cluster centre) and the membership degree of an element to the cluster is decreasing with increasing distance to the cluster centre.

To minimize elements with a small distance to the cluster it should be assigned a high membership level whereas elements with larger distances should have low membership levels. A clustering algorithm begins with a random initialization and updates the membership levels and the prototype in an iterative procedure. 


\subsection{Fuzzy Expert Systems}

Expert systems (introduced by Feigenbaum) are prolific examples within the wide scope of artificial intelligence as in [Russell and Norvig 2003] explained. Expert systems are knowledge-driven systems that can form conclusions based on knowledge on a particular field. The knowledge is represented by 'if-then' rules. By applying consequences on the stated rules, expert systems may deduce optimal decisions.

The major challenge is to commute the knowledge of subject matter experts into 'if-then' rules which are as exact as possible even given that the human representation of the knowledge cannot be well-defined determined. This downside is hurdled by usher fuzzy rules as exemplified in [Grekovs 2002].

Fuzzy rules are a collection of linguistic statements that describe how to make a decision regarding classifying an input or controlling an output:

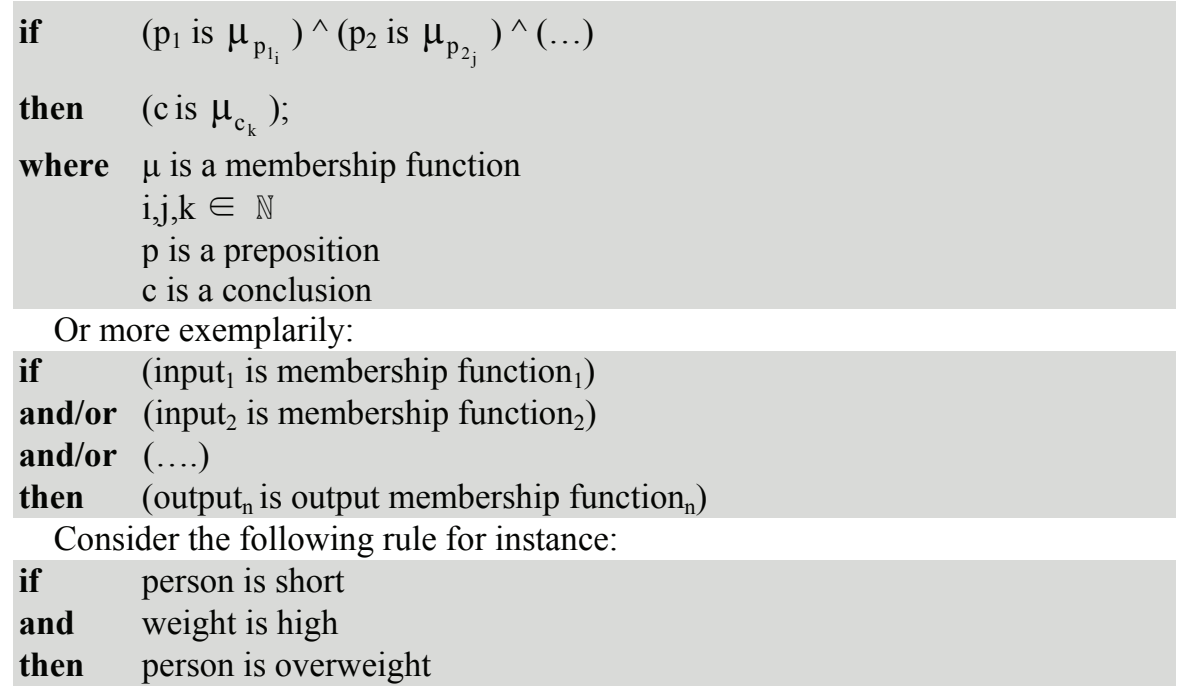

There would have to be membership functions that define what we mean by

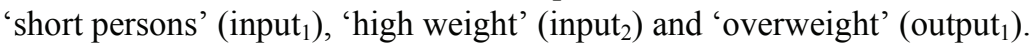

The process of taking an input such as "size" and processing it through a membership function to decide what 'short' means is stated fuzzification. The principle at that is once more to map the inputs from a set to values [0..1] using a set of input membership functions.

Thus fuzzy expert systems are usually involved when processes cannot be described by exact algorithms or when these processes are difficult to model with conventional mathematical models. 


\section{Challenges and Related Components}

This section intends to introduce the faced challenges and their related components. Section 3.1 clarifies fundamental challenges to be outgrown in the Internet of Things applications. Section 3.2 highlights the semantic homes and environments. Section 3.3 discusses necessary sensing and data provision infrastructure.

\subsection{Fundamental challenges}

Coming to a new unknown public place like a railway or underground station, airport, hospital, mall, industrial facility, corporate office, university campus all of us can remember trying to quickly find the right location, information desk, directional hint and other service availability information. Environments with quickly changing geophysical parameters like hospitals, care houses, logistic units, military and production line facilities are other problematic control domains.

Static information assistance systems related to mass produced products became a reality by the introduction of bar codes. Using modern data gathering technologies like RFID, NFC and WSN, it is possible and rational to associate various real-world entities with personalized dynamic content from weblogs, social networks, folksonomies, news feeds, location and condition tracking systems and so forth.

Semantically relevant suggestions to recognized intentions can be delivered to the user in many ways, starting from simple visualization hints, to enhanced human-machine interface influences directly on human action as in [Stapelkamp 2007; Sears and Jacko 2007] illustrated. Embedded devices for implementation of such systems will vary from the everyday smart phone to newly developed augmented reality systems.

\subsection{Semantic spaces: home and environment}

According to scientific studies, the average urban human spends about 80 to $90 \%$ of his time indoors. Buildings, houses, public places, industrial and military facilities, and even private and/or public transportation allow mankind to penetrate into all of our planet's places despite a variety of external conditions. The main indoor environment metagoals of security, safety, comfort and energy-efficiency have been implied since the very beginning of civilization. However, the approaches to pursue them are mainly restricted by the technological level available. The Internet technologies are increasingly and widely distributed today, giving us an opportunity to consider, evaluate, process and optimize the semantics behind previously developed indoor service data and information. The semantic home and environment is the concept of "thinking ambient intelligence" that is aware of its 
inhabitants (i.e. humans, animals, robots and smart objects). The extension of previously developed services to include semantically defined raw data and processed information lets emerging semantic-aware systems form (better) deductions about occurring events that affect the inhabitants. The use of multi-modal analysis of real-world sensor data and information from local and remote sites of interest is one important but challenging requirement to deducing the meaning behind these events. The deductive process is not trivial. Human actions, behaviors, habits, thoughts, intentions, emotions and health conditions are the key factors that have to be considered while tackling semantic home and environment systems, since they define event context. Context awareness is a fundamental component for informational assistance and intelligent environment behavior systems, on one hand requiring constant intelligence rule updates as in [Driver et al. 2007] illustrated. On the other hand the replayed scenarios are usually accompanied by a certain precondition sets such as: time, location, nearby resources, nearby inhabitants, action sequence, general sensory conditions such as weather, network status, service status, and others; that help in the deduction of context.

Looking from the conceptual point of view, true informational assistance is hardly imaginable without bijectional correspondence between real world and the informational model of reality. Hence, bidirectional communication methods between chip-enabled real physical objects and their informational copy are another necessary component of the ambient intelligence needed here. Following the requirements, the concepts of the Internet of Things have recently made an impressive step towards implementing the one-to-one world-to-model paradigm.

\subsection{Sensing Infrastructure and Data Provision}

The sensing infrastructure plays an important role in the provision of input data and processed information. Sensing infrastructures have variable complexity depending on the building or environment that they are installed in. For example, within a large building the data travels from disparate sensor platforms within the individual or linked buildings of different domains and authorities that were installed at different times by different vendors, with several grades of access rights and network isolation challenges to be considered along the way, via parallel subnetworks within the same building or environment. Sensing devices across the different networks vary in design sophistication in terms of connectivity, memory, mobility, energy budget and processing power; the extraction of data symbols from devices with various capabilities often incurs practical difficulties. In terms of sophistication, there are many simple battery-powered wireless devices that can be placed around a location to broadcast the current environmental condition at a periodic rate without local storage, but only few devices are designed with costly but powerful mechanisms for processing data locally, adapting their filters for noisy raw data, or supporting some query processing system for requesting data from a declarative database that is on the sensor node. Examples of sensor data spans 
across multiple domains: classical physics measurements and area measurements for ambient light, presence, temperature, infrared, absolute and relative humidity, strain; building automation data, current and voltage measurement, state of a switch or dimmer for light or blinds, smoke and fire detection system status, alarm and perimeter security status, audio-video feeds, thermostat value and setting, current weather conditions including barometric pressure and wind speed, location in localization system; industrial applications in machine health and resource plant monitoring; network availability and load of server and infrastructure systems, network security status and important changes; active and passive radio-frequency identification nodes and tag data; and many other specialized sensors and also actuators built for specific tasks.

For less sophisticated sensor devices, the data is not stored locally, but is instead transmitted across the network to a central collection point, whereas more sophisticated devices can store a considerable number of data points locally. The tradeoff of sophistication balances the cost of low-processing easy-to-move battery-powered devices capable to run for several sequential years against those of more sophisticated processing nodes that can be directly queried. Less sophisticated sensors can also use simpler, more optimized and occasionally less standardized protocols by comparison to more widespread and complex protocols such as IPv6 that allow higher interactivity in more sophisticated nodes; herein there is a challenge mitigated by an intermediary that translates the traffic to a more sophisticated protocol or stores it directly at a collection point of the particular sensing platform. These intermediate processing layers may make fuller use of individual sensor functionality than an abstraction layer does when trying to gather data through various application-level and lower protocols. There are two challenges here: to provide robustness for lost and noisy raw data and to expose the data in a way useful to information consumers. Services can expose the data in a sensing infrastructure such that a client may quickly and directly make a query with respect to a variety of contextual conditions (with heavy weight placed on location) provided by the client on what appears as one contiguous data unit, without the challenge associated with slowly querying and aggregating data from disparate sensors across the location.

Data collected into one or more query-able stores or a distributed database must be represented as data views for both public guests and elevated-privilege security-maintenance end-user clients in a standardized way that the client expects, such as using XML and other Web 2.0 and Semantic Web technologies that can expose this data using widespread standards. Here, a layer of middleware is useful in translating the data to a common standard, e.g. SPARQL, understood by a querying client newly connected to the building's middleware. Beyond basic services, additional middleware components can make available more complex services such as services that include heavily pre-processed data that includes data beyond current conditions but also forecasts trends based on data mining historical data against currently developing conditions. Between basic and complex services, a variety of data is expected to be available to the client. 
Sensor data and related services need not be restricted to a local area, even though post-processed values are cached or stored locally; the data from these distributed databases can be exposed onto the Internet of Things to be made available to a sensing infrastructure of a wider area for remote data queries of clients not yet arrived at the physical destination. This model is similar to that of the information provided by the growing number of Semantic Web services for weather forecasts, traffic forecasts, train schedules, product ratings, flight costs, outdoor and indoor directions and maps are available today and the shop sales, and other information of tomorrow; and data extracted from known and well-structured older Web 2.0 documents also into a formal knowledge concept representation. An important consideration for a resource-constrained mobile device is to consider its bandwidth when providing it a remote view of the data.

When the data is queried, an important but overlooked challenge in providing a result is to define a meaningful data structure that acts as a transport container: a structure that can define the semantic of the data being transported. Here it is obviously preferable that semantic-tagged data uses an exportable data structure model format that is componentized, standardized and commonly accepted in order to avoid redundancy in re-interpreting, or translating between other data models representing data with the same underlying semantics. Thus, whether it is simple raw data in a known standard format, e.g. SensorML, or information processed or extracted from data by some high-level transformation, any service that shares this data for export also exposes a semantic model with commonly-understood components when the response to a query is intended to be exchangeable.

\section{System Concept Description}

This section characterizes the system concept. Section 4.1 reveals an innovative information retrieval approach for future searches. Section 4.2 illustrates building blocks of the Prometheus framework. Based on ontologies, section 4.3 shows in brief, how to train the intelligent environment itself the semantic of human-used terms. Section 4.4 presents retrieved semantic data. And finally, section 4.5 discusses human-environment and human-building interaction.

\subsection{Towards an innovative information retrieval method}

The data is no longer produced by humans alone but more and more by sensors as well. As in [O'Reilly and Battelle 2009] conceptualized, today's cameras and phones are mutated into artificial ears and eyes like a sort of "sixth sense" applications. Sensors for motion and location provide continual detail where someone is, what one is looking at and how fast and in which direction one is moving. Data can be gathered and offered on a real time basis. In order to arrange these loose 
data, they need to be collected from adequate and trustworthy sources.

For this purpose information retrieval (IR), which establishes the retrieval of information from an object such as a document as outlined in [Baeza-Yates and Ribeiro-Neto 2010], comes in. In view of this, information retrieval represents the entire searching science for documents, for information within documents and for metadata about documents, as well as that of searching databases, the WWW, and other sources. At first software agents of a certain type (WWW, HTTP protocol) are instructed to collect documents in preparation for classification; in the simple example of a Web-page with natural language and images a web crawler begins at a trusted starting point and crawls along hyperlinks for each hyperlink determining the credibility and link value by use of a page ranking algorithm like HITS; and this data is reused to order search query results later. By data clustering documents can be automatically grouped into classes. Fuzzy representations are useful to handle the imprecision resulting from the automatic interpretation of extracted content meanings. The resulting interpretation is storable according to a defined domain ontology. The semantics extracted from HTML content are imprecise since they are roughly guessed when extracted and not well-defined; and the same for other text-based and multimedia objects. Semi-structured XML data is better machine readable since it is syntactically defined, but tag definitions provided by user groups are application specific and mostly ambiguous. Not so with the last example: documents tagged in representation languages designed for the Semantic Web, such as emerging RDF/S or DAML+ OIL, include formal semantics, defined rules, and an ontology vocabulary (for various types of ontologies, including service ontology) that create a well-structured machine-readable document; a significant improvement in classification. The second significant part of information retrieval is in the decision making expert systems that first contextually search for queried terms and second provide a ranked ordered list of suggested related terms of nearby clusters and other conditionals.

Generally, information retrieval systems are used to diminish what is called information overload. On the basis of a fully automated ontology with the aid of different sensors for context the IR-located information becomes arranged in a helpful manner for the user in his preferences and circumstances (e.g. location, connection speed, etc.) expanding the query beyond only the limited set of terms that describes the user need.

Paraphrased in [Marsland 2009], AI is the intelligence of machines that perceives its environment and takes actions which maximize its chances of success. A major focus there is to train the system to recognize patterns and make intelligent decisions based on this data without human intervention. However, the term of AI goes beyond the human intelligence limits and contains computational, memory and solving abilities and properties that humans do not possess. 


\subsection{The Prometheus framework}

Prometheus is a software/hardware information retrieval data processing system for the provision of the most relevant context aware information. The system uses fuzzy logic to construct the term ontology based on sensor and network distributed data. The core of Prometheus is a distributed cognitive and decision making software framework meant to be flexibly usable by humans and other software/hardware services and systems.

Functional components of Prometheus include:

- Data input subsystem gathering sensor data and moving it from sensors to between middleware and databases

- Cognitive ability subsystem implemented using several approaches from cognitive sciences (i.e. symbolic, static, behavioral, emotional) using multiple sensors data analysis capable of hierarchical activities recognition for living with predictive profiles

- Context-aware decision-making subsystem based on ontology representation and fuzzy expert systems

- Interaction subsystem with multiple machine-to-machine and humanto-machine interfaces yet unified with a backend

- Adaptable to environment communication framework allowing automatic transparent data commutation between (upcoming) WBAN, WPAN, WLAN, and Internet

Several significant impacts of Prometheus include but are not limited to:

- Increased ability for context prediction and accountability

- Diminished information overload by the context-relevant data filtering

- Workflow optimization in team and innovative project environments

- Increased energy efficiency, comfort and security of human spaces

\subsection{Ontologies and information retrieval for semantic homes and environments}

Looking at implementation, the information retrieval service is primary based on fuzzy expert systems with help of weak term ontology. Ontology is, following Gruber, a "formal, explicit specification of a shared conceptualization", that is a formal notation of a concept set within a domain and the relationships between those concepts as in [Gruber 1993] exemplified. An ontology is often needed to reason about the properties of a domain and can be used to define a field.

In [Portmann and Meier 2010] it is shown how folksonomies' subjacent tags can be harvested. To this Portmann and Meier apply particular metrics such as the Jaccard coefficient in order to meter the proximity between tags. The ontology can then be compiled without human intervention on basis of fuzzy clustering algo- 
rithms. On the basis of this ontology a meaning, or semantic, can be deduced.

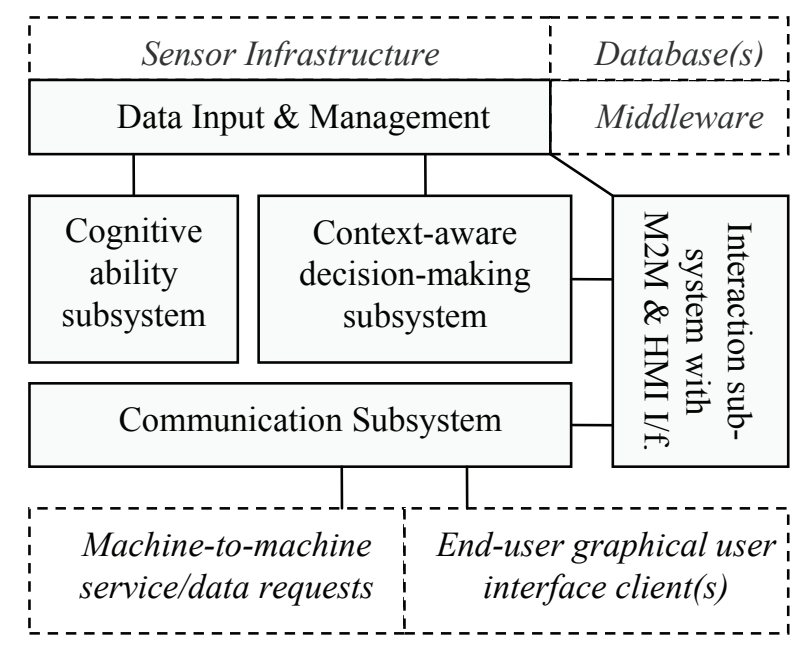

Fig. 2 Components of Prometheus framework

The Prometheus object and service search concept is based on a previously fuzzy-built and constantly automatically updated ontology, enriched with inferences from fuzzy expert systems, in order to arrive to satisfactory solutions. Fuzzy expert systems are fitting instruments for this kind of reasoning.

The interconnection between terms in the ontology allows the intelligent house to associate the user behavior and intentions with physical, economical and social parameters of the environment.

Depending on past user behavior and current context-relevant information, the Prometheus framework adaptively learns from each individual user. Behavior analysis requires storage of goals, actions, conditions and results made previously by the user and achieved as a user profile. Synthesized from this semantic description of the user prediction-based behavior assistance can be provided on the basis of fuzzy expert systems by the recognition of personal patterns from frequently repeated operations at newly visited unknown environments.

The goal of such assistance is to keep the confidence and comfort at an expected level for people dealing with unknown environments. However, every new environment will most likely not contain an exact copy of previously used objects, services and processes. In this case the most logical solution is to find objects with similar functionality and to inform the user about differences and optimal ways to access them. Moreover, informational assistance can be given directly at the "thinking event" moment, not later when the focus has already been switched to another topic. 


\subsection{Retrieved semantic data}

Enhancing the system with external data can not only improve usability but also bring an added value in a form of new location-based services as refer to in [Tsetsos et al. 2006]. Adapted from an ontology to help an end-user to get along in an unknown environment, the Prometheus framework draws on, in different dimensions available, input data such as Internet data (e.g. train connections, ratings, directions, indoor plots, taxonomies) and diverse surrounding sensor data (e.g. precise position, weather conditions, traffic jams, local accidents, door or elevator malfunctions, power losses, water and heating shut offs) to present intelligent suggestions (e.g. fastest directions to a certain destination, the next train station, connections, prices, ratings, pictures, descriptions of a specific product).

Additionally, for the moment "not relevant" data like changing weather conditions while shopping is frequently useful right at the next moment because it can influence the user's (buying) preferences and decisions.

Data to be shown has to be chosen according to the user role, current semantic context and current goal set. Moreover, the group of people has to be also considered while designing the communication dataset. Requirements like people group-oriented data availability, people collaboration encouragement, environment adaptability, accountability, security and privacy shape the dataset.

\subsection{Environment and building interaction}

To reveal relevant data, the Prometheus framework decides under given circumstances how to interact optimally. Using human-computer interaction the building can adapt by changing visual, audio, thermal, humidity, pressure or other physical environment parameters depending on the mood of a tenant.

The search query itself is usually considered as an interface between the user and a search engine. Prometheus is extending the understanding of search queries towards user goals, intentions and behavior. The system does not simply provide the string search box with historically based associative suggestions, but also keeps in mind the semantic context of the user or client system.

The way the semantic environment interacts with a human strongly overlaps with studies from the human-computer-interaction field, as for example the basic interface simplicity requirement. Also, display design principles remain the same.

However, different semantic system parameters define specific functional constraints leading to the terms of human-building-interaction and humanenvironment-interaction. The relevant data will be presented in a for the user appropriate way adapted for the subjacent hardware.

This work associates buildings with being indoors while environment with being outdoors. The main difference between these two types of human interaction is characterized by different impact factors of the common life-value parameters. This parameter list is not hard-fixed for every person. It is mainly provided for a 
demonstrative purpose. Table 1 contains the parameter impact factors belonging to the range $[1 \ldots 5]$. The highest value corresponds to the highest impact factor.

Table 1: Simplified Example of Parameters Impact

\begin{tabular}{|c|c|c|}
\hline \multirow[t]{2}{*}{ Parameter } & \multicolumn{2}{|c|}{ Impact factor on an action } \\
\hline & Indoors & Outdoors \\
\hline Weather conditions & 2 & 5 \\
\hline Customization & 4 & 1 \\
\hline Location & 3 & 4 \\
\hline Security & 4 & 4 \\
\hline Comfort & 5 & 2 \\
\hline Safety & 4 & 4 \\
\hline Time & 2 & 5 \\
\hline Cost & 4 & 3 \\
\hline
\end{tabular}

We observe that weather conditions and time of day have much stronger influence on human actions outdoor than indoor. The human action impact of customization and comfort is in opposite relatively little outdoor and significantly high inside of the buildings. Security, safety, location and cost are equally important for human interaction independent of the environment.

\section{Practical Applications}

The previous more theoretically oriented sections showed stimuli for this section. As an example, a personal digital assistant concept for embedded devices like smart phones and house appliances is presented.

For improved support a future system, like a smart phone, should help people in the same way that present personal digital assistants do. Based on the proposed ontology the digital assistant could educe feasible suggestions for the user. To come up with such suggestions, a future smart phone's built-in personal digital assistant, here referenced as a part of Prometheus system, has to learn first from the elapsed user's environmental data. Then it would be possible to pick (for the user) the best solution based on the fuzzy expert systems method.

A major challenge hereby is to teach the digital assistant appropriate solutions. Therefore the user has sometimes to interact with the personal digital assistant. As known from supervised learning, the user should correct potential personal digital assistants misbehavior. Learning inputs can also come from environmental sensor data in which no user interaction is required.

The simplest example is based on the current (indoor or outdoor) location data 
and needs of the user. For example, the optimal solution for a query on "shoes" will include costs, time, warranty conditions, the (indoor or outdoor) way, and connection for public transportation. Figure 3 shows the particular possible use case while utilizing the Prometheus framework components.

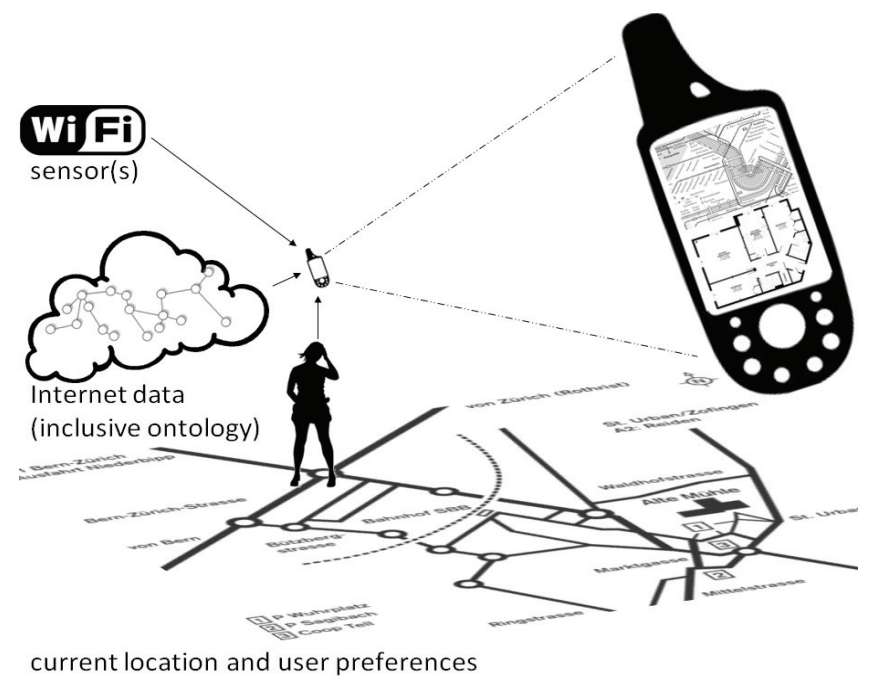

Fig. 3 The Internet-, sensor- and user-preference-data are expected come up with bright solutions for the user.

\section{Conclusions and Outlook}

After a short introduction into the fuzzy set theory and its applications, this paper presented the main foreseen challenges to be faced by shaping semantics behind the existing living infrastructure. The presented approach of the Prometheus framework depicts a vision towards a solution of these challenges with the novel approach based on fuzzy set theory in the rapidly developing area of the Internet of Things. Furthermore towards the dataset considerations for semantics implementation particular attention was given to the new terms of human-building and human-environment interaction.

Further subjacent studies on the issue of appropriate outcome-rules for the fuzzy expert system will be needed. It might be that the proposed approach could be improved by taking into account other machine-learning strategies. At the moment Prometheus is thought to be based on fuzzy rules, but in the future it is not limited to only this.

Another point is a possible enhancement of the fuzzy-built ontology towards integrating further sensor data as for example intelligent clothes. Wearable compu- 
ting is a vigorous research topic, containing user interface design-, augmented reality-, pattern recognition-, use of wearable's for specific applications or disabilities, electronic textiles and fashion design studies.

The CEESAR-iHomeLab is working on one hand on forming a scientific community for cutting-edge international research projects in the area of ambient intelligence, human-building interaction, user behavior analysis, and assisted living and on the other hand; the Research Center FMsquare implements the ideas of fuzzy methods to various scope of applications, and for this reason both research centers appreciate cooperation with researchers and practitioners.

\title{
Acknowledgment
}

We sincerely thank our colleagues from CEESAR and its iHomeLab (http://www.iHomeLab.ch/) project at the Lucerne University of Applied Sciences, who always helped with word and deed. Furthermore, a special thank goes to the Fuzzy Marketing Methods Research Center (www.FMsquare.org) of the University of Fribourg for contributing valuable thoughts.

\section{References}

\author{
[Azuma et al. 2001] \\ Azuma, R., Baillot, Y., Behringer, R., Feiner, S., and B. MacIntyre. Recent Advances in Augmented \\ Reality. IEEE Computer Graphics and Applications, Volume 21, Issue 6, pp. 34 - 47, November 2001. \\ [Baeza-Yates and Ribeiro-Neto 2010] \\ Baeza-Yates, R., Ribeiro-Neto, B. (1999). Modern Information Retrieval. ACM press, Essex, 1999. \\ [Bishop 2008] \\ Bishop, C. M. Pattern Recognition and Machine Learning. Springer, Berlin, 2008. \\ [Driver et al. 2007] \\ Driver, C., Linehan, E., Spence, M., Tsang, S. L., Chan L. and Clarke, S. Facilitating Dynamic Sche- \\ dules for Healthcare Professionals. Pervasive Health Conference and Workshop, pp. 1 - 10, 2007. \\ [Gruber 1993] \\ Gruber, T. R. A Translation Approach to Portable Ontology Specifications. Knowledge Systems La- \\ boratory, Technical Report KSL 92-71, pages 199-220, April 1993. \\ [Grekovs 2002] \\ Grekovs, R. Methods of Fuzzy Pattern Recognition. Proceedings of Riga Technical University, 2002. \\ [IoT: Summary] \\ International Telecommunication Union (2005). The Internet of Things. Executive Summary.
}


[Kasabov 1996]

N. K. Kasabov Foundations of Neural Networks, Fuzzy Systems, and Knowledge Engineering. MIT Press, Cambridge, 1996.

[Marsland 2009]

Marsland, St., Machine Learning. An Algorithmic Perspective, CRC Press, 2009.

[O’Reilly and Battelle 2009]

O'Reilly, T., Battelle, J. Web Squared: Web 2.0 Five Years On. Web 2.0 Summit, O’Reilly Media, Inc, pages 1-13, 2009.

[Portmann and Meier 2010]

Portmann, E., Meier, A., "A Fuzzy Grassroots Ontology for improving Weblog Extraction", In: Journal of Digital Information Management, pp. 276-284, Chennai, India, August 2010.

[Russell and Norvig 2003]

Russell, S. J., Norvig, Peter, Artificial Intelligence: A Modern Approach (2nd ed.), Prentice Hall, 2003.

[Stapelkamp 2007]

Stapelkamp, T. Screen- und Interfacedesign. Springer Science, Berlin 2007, ISBN 3-540-32949-8

[Sears and Jacko 2007]

Sears, A., Jacko, J. A. Handbook for Human Computer Interaction .CRC Press. ISBN 0-8058-5870-9

[Tsetsos et al. 2006]

Tsetsos, V.,Anagnostopoulos, C., Kikiras, P., Hadjiefthymiades, S. Semantically enriched navigation for indoor environments. Int. Journal of Web/Grid Services, Volume 2, December 2006, pp. 453-478.

[Zadeh 1965]

Zadeh, L. A. "Fuzzy Sets”, Information and Control, 8, 1965, pp. 338-353. 